\title{
The Airbus A320 Family Fan Cowl Door Safety Modification: A Human Factors Scenario Analysis
}

\author{
Kyriakos I Kourousis $^{1}$, Anna V Chatzi ${ }^{2}$, Ioannis Giannopoulos ${ }^{3}$
}

\author{
${ }^{1}$ School of Engineering, University of Limerick, Limerick, Ireland \\ ${ }^{2}$ School of Commerce, University of Southern Queensland, USQ Toowoomba Campus, QLD, \\ Australia. \\ ${ }^{3}$ School of Aerospace, Transport and Manufacturing, Cranfield University, Cranfield, \\ Bedfordshire, United Kingdom.
}

\begin{abstract}
Purpose - The Airbus A320 family engine fan cowl doors safety issue is known to the industry for almost 18 years, however it has not been addressed adequately by the aircraft manufacturer and the various operators and regulating authorities. This brief case study paper examines in a systematic way the possible operational and safety implications of a new modification on the engine fan cowl doors.
\end{abstract}

Design/methodology/approach - An array of error-prone scenarios are presented and analysed under the prism of human factors in a non-exhaustive qualitative scenario analysis.

Findings - All examined scenarios are considered more or less probable. A number of accident prevention solutions are proposed for each of the scenario examined, in view of the acceptance and implementation of this modification by operators.

Research limitations/implications - Since these scenarios are neither exhaustive, nor have been tested/validated in actual aircraft maintenance practice further analysis is necessary. A substantial follow up survey should take place, which should include a wider array of scenarios. This would allow obtaining the necessary data for a quantitative (statistical) analysis.

Practical implications - This case study identifies issues in relation to this modification, introduced by Airbus and the European Aviation Safety Agency (EASA), which may prove problematic from the point of view of safety effectiveness and disruption of operations.

Originality/value - This case study examines a long-standing aviation safety issue and the implications of a solution proposed by the aircraft manufacturer and adopted by EASA. This can be useful in increasing the awareness around these issues and highlight the importance of a human-centric and scenario-based design of engineering modifications towards minimising error in aircraft technical operations.

\section{Keywords}

Human factors, aviation maintenance, safety, airworthiness, accident prevention 


\section{Introduction}

Several Airbus A320 family engine fan cowl door (FCD) (Figure 1) losses have occurred in the past due to uninspected unlocked situations that have occurred in service (AAIB, 2015). This issue is known to the industry for almost 18 years, however it has not been addressed adequately by the aircraft manufacturer (Airbus) and the various operators and regulating authorities. Similar issues have been faced in the past with other aircraft types, such as the ATR-42 (AEAT, 2002).

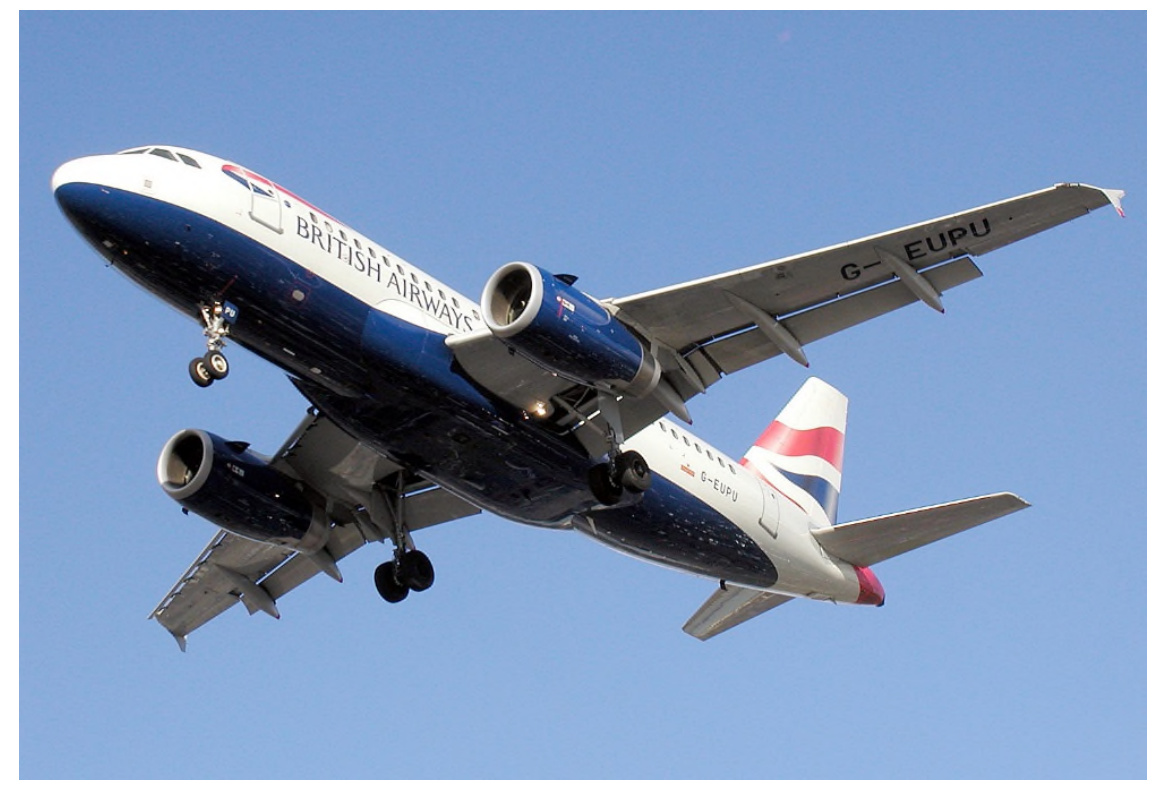

Figure. 1 A British Airways Airbus A319-100, where the (blue-painted) fan cowl doors (FCDs) surrounding the engines are shown (photograph by Adrian Pingstone). [https://commons.wikimedia.org/wiki/File:Britaw.a319-100.g-eupu.arp.jpg. Public domain].

A historical overview offers an interesting insight on the FCD safety issue, by looking at the preceding modifications (manufacturers' SBs), issued EASA and Federal Aviation Authority (FAA) ADs and FAA proposed rulemaking documents (Notice for Proposed Rule Making, NPRM) (Figure 2). What stems from this brief examination is that following an activity in the early 2000's, the issue was practically silenced (from the standpoint of redesign and safety regulation) for 12 years, despite the ongoing incidents. Airbus, as the aircraft design approval holder, has re-opened the investigation and mitigation of this safety issue in reaction to an accident investigation report released in 2015 by the United Kingdom (UK) Air Accidents Investigation Branch (AAIB). 


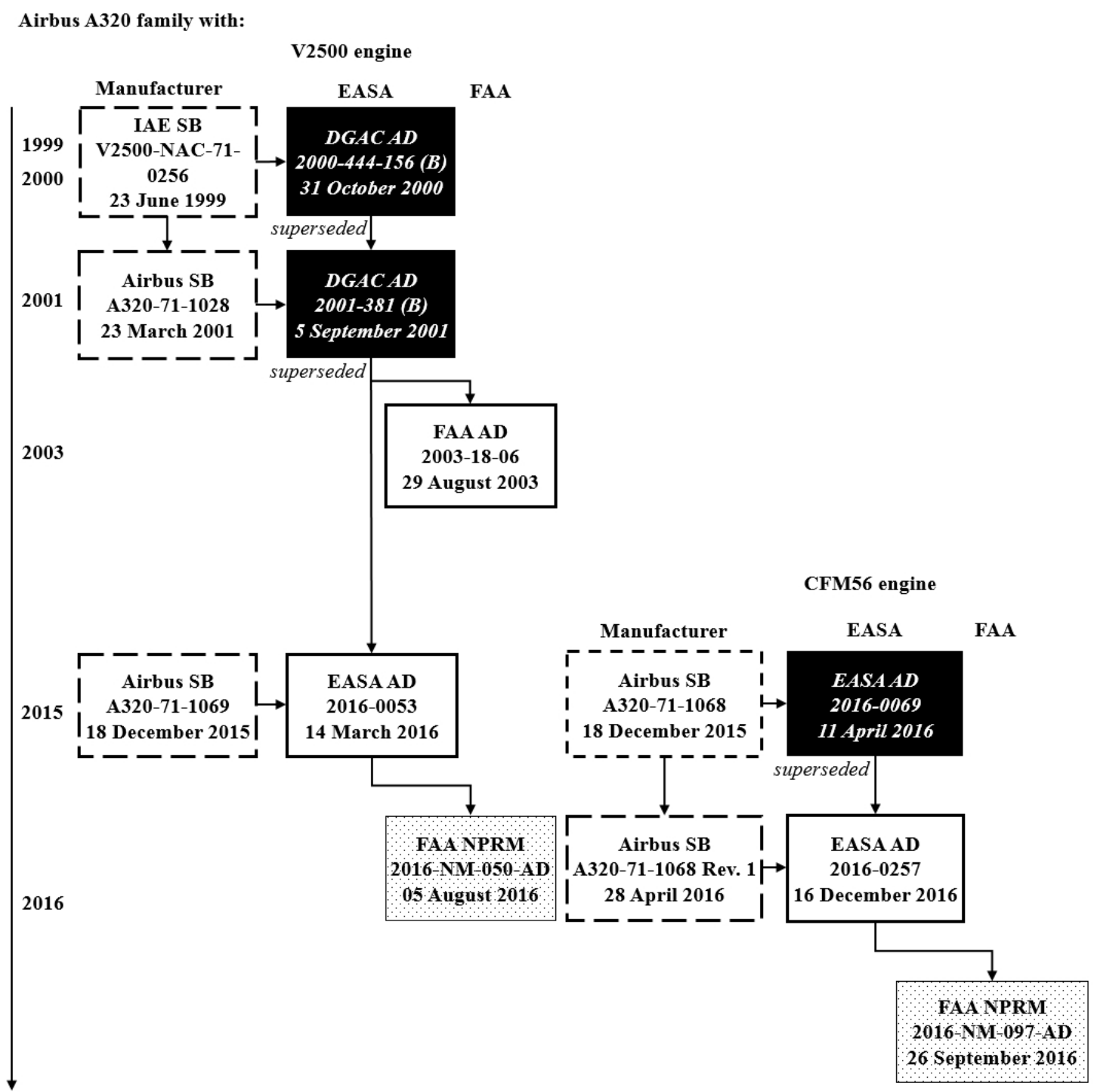

Figure 2. A historical overview of the manufacturers' and regulating authorities' (EASA, FAA) actions on the Airbus A320 family engine FCD safety issue. Note: DGAC refers to the French aviation regulator ('Direction Générale de l'Aviation Civile').

In particular, it was a double FCD loss from a British Airways Airbus A319 in 2013 (Figure 3) that has led to the escalation of this issue, following the release of the 2015 AIB accident investigation report (AAIB, 2015). Airbus, in an attempt to address the issue permanently, proceeded in redesigning the FCD locking arrangement and control philosophy (Airbus, 2015a; Airbus, 2015b), which were subsequently adopted by the European Aviation Safety Agency (EASA), in 2015 and 2016, as Airworthiness Directives (ADs) (EASA, 2016c; EASA, 2016d). Both EASA ADs are currently under consideration by FAA (FAA, 2016a; FAA, 2016b). 


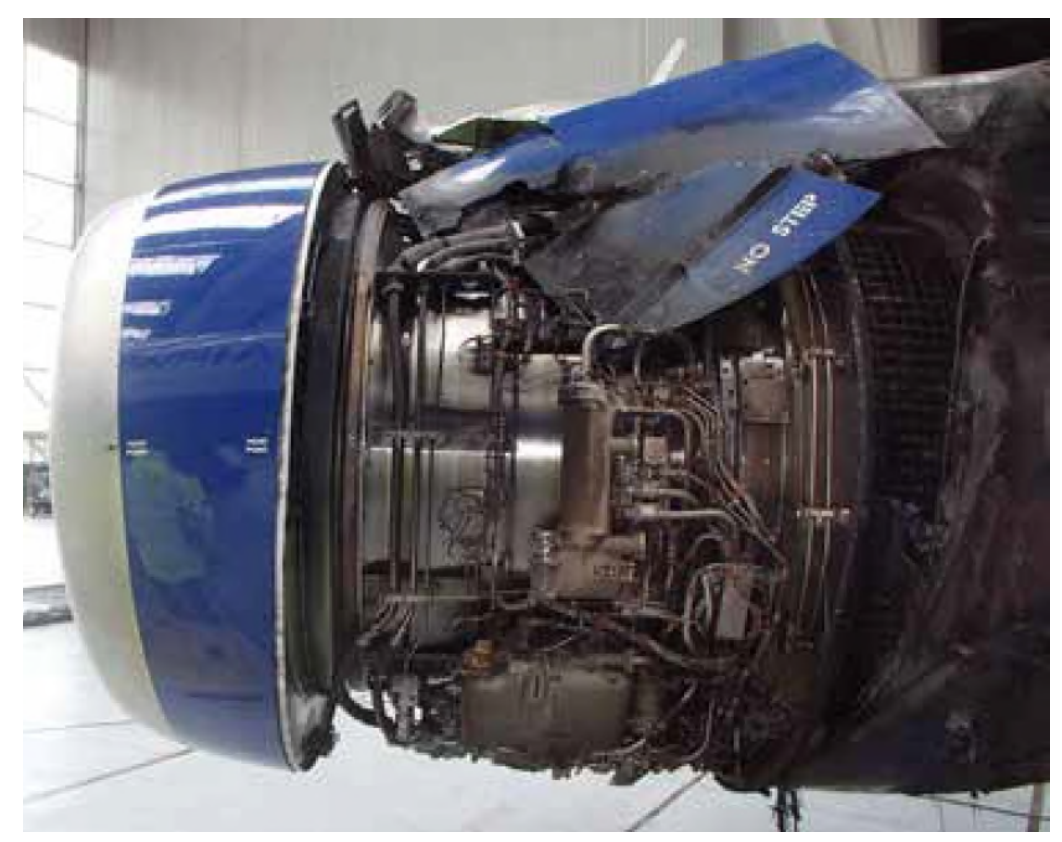

Figure 3. Remaining parts of the right-hand engine inboard FCD of the British Airways Airbus A319-131 G-EUOE following the 24 May 2013 accident (photograph reproduced from the AAIB Aircraft Accident Report 1/2015 (AAIB, 2015)).

The 2016 EASA ADs and the relevant Airbus Service Bulletins (SBs) describe the modification that the aircraft operators has to implement on all affected models of the Airbus A320 family (A318/319/320/321) fitted with the IAE V2500 and CFM56 engines. The main features introduced by this modification are (EASA, 2016c; EASA, 2016d):

- A new FCD front latch which locks/unlocks with a use of a specific key (the two other latches remain unchanged) (Figure 4). This key cannot be removed once the latch is unlocked.

- A new locking/unlocking key for the FCD front latch with a ('remove before flight') flag fitted on it (Figure 4). The flag increases the visibility-detectability of an unlatched condition, since the key-flag assembly is attached to the latch as long as it remains in the open position.

- A key keeper assembly at a designated storage area in the cockpit, where the key and the ('remove before flight') flag assembly are kept when once the FCD is closed.

- Aircraft Maintenance Manual (AMM) adaptation, to include provisions for a logbook entry requirement when opening/closing the FCDs is performed, as a way to assist communication and raise awareness over the matter. 


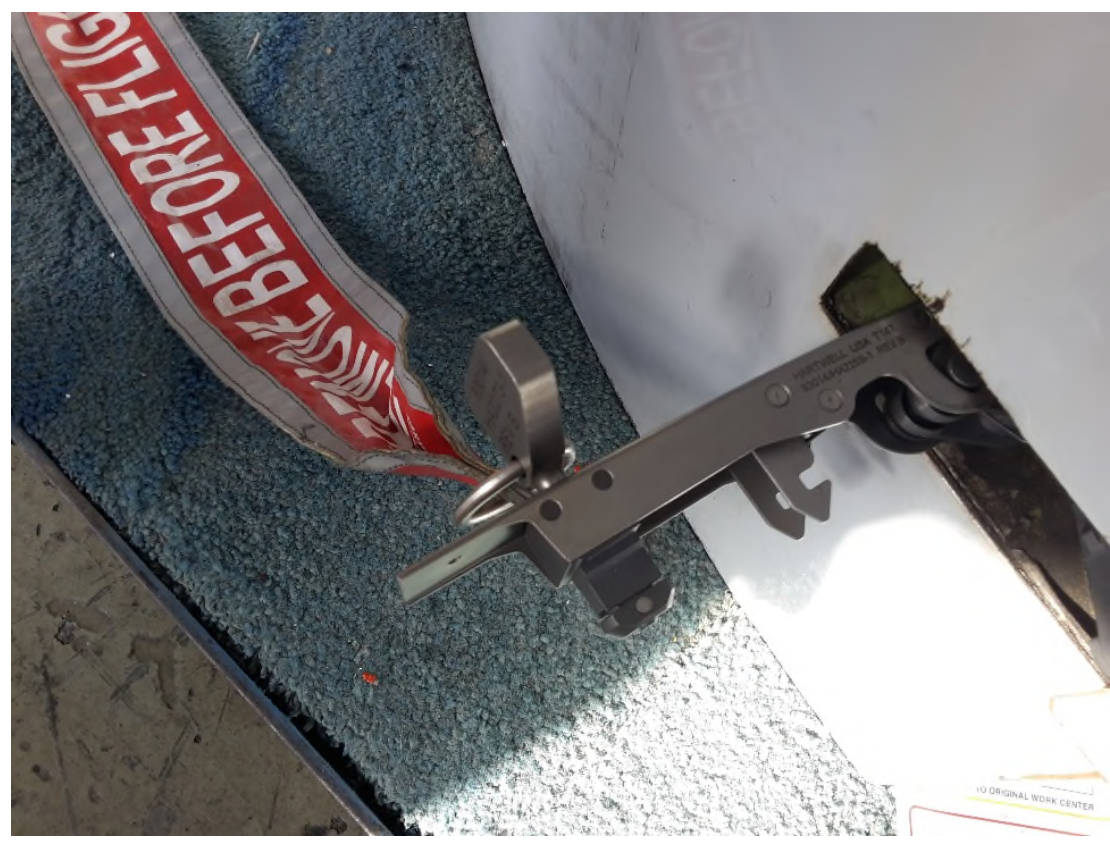

Figure. 4 Modified A320 family engine FCD with new latch and key - 'remove before flight' flag assembly.

However, as part of the EASA ADs' consultation process (conducted prior to their issue), a number of major operators (United Airlines, American Airlines, All Nippon Airways, Air Canada) have expressed reservations on the effectiveness of the Airbus redesign, on the basis of human factors issues, potential financial impact on operations and implementation cost (EASA, 2016a; EASA, 2016b). For example, United Airlines, in their comments to EASA (EASA, 2016a) argued that the implementation of another visual cue does not guarantee that the people involved will not miss it, unless they are careful and attentive. In the same response, United Airlines highlighted that dual sign-off for the FCD closure and other steps they have introduced in their operational procedures (towards increasing the awareness of the technical staff) have proved to be successful in addressing human factor related issues. In particular, United Airlines has not had any incidents occurring since the introduction of these, human factor focused, measures in 2006. Similarly, Air Canada supported the suitability and effectiveness of the dual sign procedure, expressing a strong negative view on the usefulness of the modification (EASA, 2016b). As Air Canada highlighted in their comments, a uniform solution approach is not likely to be effective, since each organisation should work towards changing the technical staff culture to address the safety issues around FCDs (EASA, 2016b). As also recorded in the (EASA, 2016a; EASA, 2016b), one may note that, in response to these comments, EASA did not make any changes in the final ADs, while they suggested that operators may apply for an Alternative Means of Compliance (AMC) to the AD, by providing data supporting their requests (for exemption from the AD).

The EASA's reasoning behind the adoption of the Airbus FCD SB is not described in the ADs. Moreover, the design principles employed by Airbus, in the development of the SB, is not known (as the SB is not publicly available). The adopted solution is considered peculiar for aviation maintenance, from the point of view of human factors, since it is not usual practice to restrict access to aircraft compartments via specific keys, rather than standard or special tools. Extensive review has failed to identify similar solutions utilised in civil aviation. 
This brief paper intends to examine and discuss in a systematic way the possible operational and safety implications that the FCD modification can have in aircraft maintenance practice.

\section{Qualitative scenario analysis, results and discussion}

Taking into account the aircraft modifications and the changes in the maintenance processes which occur from the EASA ADs, we have examined and identified steps in the new procedures that may prove problematic from the point of view of safety effectiveness (increase errors or lead to deviations from safe practice) and disruption of operations (create delays/obstructions in aircraft dispatch/maintenance). In particular, an array of error-prone scenarios are presented and analysed under the prism of the human element.

The scenarios were developed conceptually by utilising the authors' $10+$ years' experience in aircraft maintenance practice (as certifying staff), design/certification of modifications and accident/incident investigation. The realism of the scenarios (steps, sequence, etc.) was also validated by consulting aircraft maintainers having prior experience on the A320 family aircraft. Thus, both the development and validation of the scenarios did not require any physical work on aircraft (or any interaction with an aircraft maintenance organisation).

Moreover, accident prevention solutions are proposed for each of the scenario examined. It is noted that within the EASA framework these recommendations are part of the existing Maintenance Resource Management (MRM) training and the EASA Part-66 and Part-145 human factors training requirements (EU, 2014). Errors related to handovers generally have more severe and dangerous consequences, as approximately half of the aircraft maintenance failures due to ineffective communication are related to the shift handover (Parke and Kanki, 2008). Debriefs which are based upon human factors considerations have the potential to enhance productivity by $20 \%-25 \%$ (Tannenbaum and Cerasoli, 2013). Effective teamwork is known to be essential in safer aviation maintenance practice (Leonard et al, 2004; Robertson, 2005; Sexton et al, 2000), mainly due to the nature of the profession (organisational structure of work, rather than individuals working in isolation). Time pressure, such as that experienced in the flight line environment, is a primer for errors (Goglia et al, 2002; Reason 2000) and, in this case, it is considered important to examine. Overall, teamwork, dual sign-offs, effective time management and request for assistance from colleagues and supervisors (whenever required) constitutes good practice in aviation MRM.

According to the FAA, MRM can also act as a training programme, as it aims to alter the technicians' attitude and perspectives in order to establish safety as their primary goal (Robertson 2005). As regularly reported in the literature, training in aviation is important and it acts beneficiary, while its design, delivery and implementation needs to be tailored to the needs of the organisation (Lappas and Kourousis, 2016; Salas et al, 2012; Taylor and Thomas, 2003). Consequently, aircraft maintenance managers should consider the training process as a proactive safety measure and actively support MRM training. It is of note that employees working within the highly regulated aviation industry, are inclined to practice more safely than in a more productive fashion (Karanikas et al, 2017). This is a strong indicator of how the 'safety over productivity' equilibrium can be positively influenced (towards safety) by regulation.

It is interesting to look at the definition of (Wiegmann,et al, 2004) on safety culture "as the shared values, beliefs, assumptions, and norms that may govern organisational decision making, 
as well as individual and group attitudes about safety". Focusing on the norms of an organisation, these set the framework within the employees are expected to think and operate (Wreathall, 1995). Therefore, if the norms contradict the organisation's safety policy, they should be revised or abolished. Any organisation, in order to action changes in culture, they have to establish effective safety communication between the various organisational and managerial levels. In aircraft maintenance training, this can include the establishment of a thorough safety training programme (Geldart et al, 2010; Hall et al, 2016).

The scenarios are not intended to be exhaustive, in terms presenting the full spectrum of combinations of actions. However, they represent a number of cases which are deemed likely to occur in service and that can have a considerable impact on safety and operations. All scenarios start from the case of a maintenance task requiring access to the area enclosed by the FCD (in the cases examined 'engine failure troubleshooting'), which is secured by the specific key (introduced with the Airbus modification/EASA ADs).

\section{FCD key in designated area}

The technician retrieves the FCD key from the designated storage area in the cockpit and inserts a logbook entry for the opening/closing of the FCD.

Scenario 1 The technician leaves the maintenance task (in the area enclosed by the FCD) for the end of the failure troubleshooting. He/she performs the maintenance task at the end of his/her shift. However, he/she does not dedicate adequate time for the maintenance task, as he/she inadvertently prioritised the FCD task [return of the key, closure of the logbook entry ('FCD closed')], in an effort to avoid the FCD is not left open. This poor practice may result in reduced maintenance quality, under stressful or very time constrained situations, since FCDrelated tasks are added to the existing workload. Prevention measures may include: putting more focus on time management techniques and requesting assistance from peer-workers/team leader in stressful/time-pressing situations.

Scenario 2 The technician performs the maintenance task straight away but leaves the key return and logbook entry closure for later. Since these steps were left for a later time, the technician either forgets completely to return the key/close the logbook entry or gets distracted near that time, having the same result. As a consequence, the aircraft release to service can be delayed, since the involved personnel (flight crew, technical staff) will have to locate the missing key and complete the FCD sign-off in the logbook.

A dual sign off practice would offer the opportunity for a confirmation check and reduce the possibility of misses and errors.

Scenario 3 The technician does not perform the maintenance task and has to pass it over to the next shift. Since these steps were left for the next shift, he/she either forgets to return the key/close the logbook entry or gets distracted to do that. In case that the shift handover is not performed properly, the FCD tasks are not completed. As a consequence, similarly to Scenario 2 , the aircraft release to service can be delayed, since the missing key has to be located and the logbook signed off.

As with Scenario 2, the dual sign off practice can mitigate this issue. Moreover, a thorough (verbal and written) shift handover would be helpful in avoiding communication gaps in relation to the FCD tasks (reducing the possibility for misses and errors). 


\section{Key missing from designated area}

The technician does not find the FCD key in the designated storage area in the cockpit.

Scenario 4 The technician attempts to find the FCD key. He/she prioritises this task over the maintenance task itself. In the case that he/she finds the key, the amount of time spent on the search does not allow him/her to focus on the maintenance task, thus this is not performed adequately.

Similarly, to Scenario 1, it would be beneficial if better time management techniques were practiced, as well as if the technician requested assistance.

Scenario 5 The technician attempts to find the FCD key, prioritising the search over the maintenance task (same as in Scenario 4). He/she does not manage to find the key, leaving the maintenance task unaccomplished. In the case that the technician is forgetful or distracted, he/she will not report the missing key, causing more delay, as other personnel in later time will repeat the search process.

Similarly to other scenarios, the dual sign off in conjunction with a robust handover process could mitigate this miss.

Scenario 6 The technician attempts to find the FCD key, prioritising the search over the maintenance task (same as in Scenario 4 and 5). He/she does not manage to find the key, therefore deciding to use his/her own key or the spare key as per the organisation's 'norm', and fills in the logbook entry ('open FCD'). After completing the maintenance task the technician is forgetful/omits or gets distracted and does not report the missing key. As with Scenario 5, this may cause a delay in the future. Moreover, using his/her own key means that this may not have the 'remove before flight' flag attached, increasing the probability of leaving the cowl door open (since this modified visual cue will be missing).

Similarly to previous scenarios, the dual sign off in conjunction with a robust handover process could mitigate this miss. In addition, a change in the organisational culture would be necessary to abolish unsafe practices in relation to established 'norms' outside the standard policies and procedures.

Scenario 7 The technician does not have the required time or attitude to attempt to find the missing key, thus he/she decides not to perform the assigned maintenance task and, for example, to move onto a different task. He/she forgets about the missing FCD key or gets distracted and does not report that. This shall cause delay in the work of the personnel who are then assigned to the maintenance task in the FCD-accessed area (as they will have to search for the missing key).

Dual sign off and in-shift/inter-shift handover would be an effective solution to avoid such situations.

\section{Graphical Representation of the Scenarios}


The sequence of the events and causes described in each of the Scenarios (1 to 7) is graphically represented in Figure 5, where all interconnections are shown. The graph illustrates characteristically the complexity of the various problematic situations that may arise out of the subject matter FCD safety modification. 


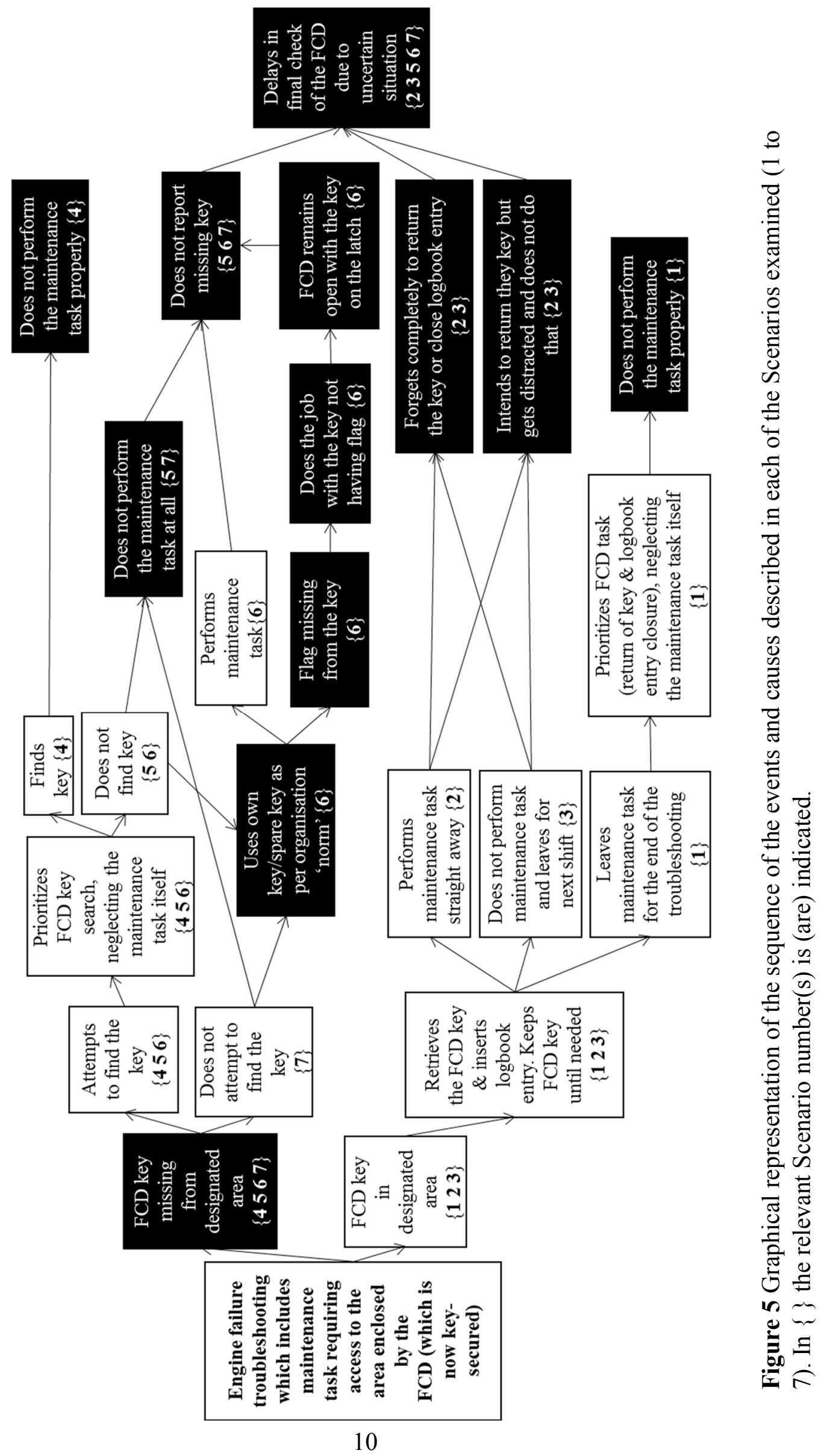


Since all scenarios are realistic they are considered more or less probable. Typically, these may be encountered by technicians working both in the line and base level aircraft maintenance environment. However, since these scenarios are neither exhaustive, nor have been tested/validated in actual aircraft maintenance practice further analysis is necessary. For that purpose, a substantial survey, which should include a wider array of scenarios, would be necessary to obtain the necessary data for a quantitative (statistical) analysis.

\section{Conclusion}

The A320 family FCD safety issue cannot be considered as a trivial issue, since it has been concerning the aviation industry over the past 18 years. It is anticipated that the Airbus modification - EASA ADs shall be able to contribute positively to the error management regarding FCD losses. However, it is important to consider the associated human attitude elements brought in with this modification, as illustrated by this qualitative scenario analysis. To this end, a list of human factors centred procedures and actions are recommended. These stem from the various scenarios, described and discussed in the previous section, and consist of all possible attitudes and responses of the technicians towards the new modifications. In summary, the recommended actions are: provision of better time management training, enhancement of communication skills, focused training, encouraging a collaborative attitude, implementation of a dual sign off procedure for the opening/closing of the FCDs, thorough verbal/written shift handover and facilitation of changes in the airline/maintenance organisation culture (where necessary). These measures are able to achieve efficiencies in procedures associated with troubleshooting in the area enclosed by the key-accessed FCD, reduce the likelihood of errors, and, most importantly, identify and suppress any safety-infringing 'norms' within operators and maintenance organisations. 


\section{References}

Air Accidents Investigation Bureau (AAIB) (2015), "Report on the accident to Airubs A31913, G-EUOE London Heathrow Airport 24 May 2013”, Aircraft Accident Investigation Report, Alderhot, Hampshire, United Kingdom, 14 July 2015.

Airbus (2015a), Service Bulletin A320-71-1068, 18 December 2015.

Airbus (2015b), Service Bulletin A320-71-1069, 18 December 2015.

Aircraft Engineering and Aerospace Technology (AEAT) (2002), “Loss of Engine Cowl Door", Vol. 74 No. 1, pp. 51-56.

European Aviation Safety Agency (EASA) (2016a), Comment Response Document on Proposed Airworthiness Directive (PAD) No. 16-009, 26 January 2016.

European Aviation Safety Agency (EASA) (2016b), Comment Response Document on Proposed Airworthiness Directive (PAD) No. 16-022, 10 February 2016.

European Aviation Safety Agency (EASA) (2016c), Airworthiness Directive (AD) 20160053, 14 March 2016.

European Aviation Safety Agency (EASA) (2016d), Airworthiness Directive (AD) 20160257, 16 December 2016.

European Union (EU) (2014), Commission Regulation No 1321/2014, On the continuing airworthiness of aircraft and aeronautical products, parts and appliances, and on the approval of organisations and personnel involved in these tasks, 26 November 2014.

Federal Aviation Authority (FAA) (2016a), Notice of Proposed Rulemaking (NPRM) 2016NM-050-AD, 5 August 2016.

Federal Aviation Authority (FAA) (2016b), Notice of Proposed Rulemaking (NPRM) 2016NM-097-AD, 26 September 2016.

Geldart, S., Lohfeld, L., Shannon, H. S., \& Smith, C. A. (2010). Organizational practices and workplace health and safety: A cross-sectional studying manufacturing companies. Safety Science, Vol. 48, pp. 562-569.

Goglia, J., Patankar, M. S., \& Taylor, J. C. (2002). Lack of error mitigation tools: The weakest link in maintaining airworthiness? Paper presented at the 55th Annual International Air Safety Seminar, Dublin, Ireland.

Hall, A., Oudyk, J., King, A., Naqvi, S., \& Lewchuk, W. (2016). Identifying knowledge activism in worker health and safety representation: A cluster analysis. American Journal of Industrial Medicine, Vol. 59, pp. 42-56.

Karanikas, N., Melis, D. J., \& Kourousis, K. I. (2017). The balance between safety and productivity and its relationship with human factors and safety awareness and communication in aircraft manufacturing. Safety and Health at Work, pp. 1-8. 
Lappas, I., \& Kourousis, K. I. (2016). Anticipating the need for new skills for the future aerospace and aviation professionals. Journal of Aerospace Technology and Management, Vol. 8, pp. 232-241.

Leonard, M., Graham, S., \& Bonacum, D. (2004). The human factor: The critical importance of effective teamwork and communication in providing safe care. Qual Saf Health Care, Vol. 13, pp. 85-90.

Parke, B., \& Kanki, B. G. (2008). Best practices in shift turnovers: Implications for reducing aviation maintenance turnover errors as revealed in ASRS reports. The International Journal of Aviation Psychology, Vol. 18, pp. 72-85.

Reason, J. (2000). Human error: Models and management. BMJ: British Medical Journal, Vol. 320, pp. 768-770.

Robertson, M. (2005). Maintenance resource management. In F. A. Authority (Ed.), Human factors guide for aviation maintenance.

Salas, E., Tannenbaum, S. I., Kraiger, K., \& Smith-Jentsch, K. A. (2012). The science of training and development in organizations: What matters in practice. Psychological Science in the Public Interest, Supplement, Vol. 13, pp. 74-101.

Sexton, J. B., Thomas, E. J., \& Helmreich, R. L. (2000). Error, stress, and teamwork in medicine and aviation: Cross sectional surveys. British Medical Journal, Vol. 320, pp. 745749.

Tannenbaum, S. I., \& Cerasoli, C. P. (2013). Do team and individual debriefs enhance performance? A meta-analysis. Human Factors, Vol. 55, pp. 231-245.

Taylor, J. C., \& Thomas III, R. L. (2003). Written communication practices as impacted by a maintenance resource management training intervention. Journal of Air Transportation, Vol. 8, pp. 69-90.

Wiegmann, D. A., Zhang, H., Von Thaden, T. L., Sharma, G., \& Gibbons, A. M. (2004).

Safety culture: An integrative review. The International Journal of Aviation Psychology, Vol. 14, pp. 117-134.

Wreathall, J. (1995). Organizational culture, behavioral norms and safety, Paper presented at the International topical meeting on safety culture in nuclear installations, Austria, Vienna. 
2018-08-18

The Airbus A320 family fan cowl door safety modification: a human factors scenario analysis

Kourousis, Kyriakos I.

Emerald

Kyriakos I. Kourousis, Anna V. Chatzi and loannis K. Giannopoulos. The Airbus A320 family fan cowl door safety modification: a human factors scenario analysis. Aircraft Engineering and Aerospace Technology, Volume 90, 2018, Issue 6, pp. 967-972

https:doi.org/10.1108/AEAT-08-2017-0191

Downloaded from Cranfield Library Services E-Repository 